\title{
Comparative Study between Two Schemes of Active-Control-Based Mechatronic Inerter
}

\author{
Lingduo $\mathrm{He}$, Yaozong Liu and Silu Han \\ College of Mechatronic Engineering and Automation, National University of Defense Technology, Hunan, China
}

\begin{abstract}
Based on force-current analogy and velocity-voltage analogy in the theory of electromechanical analogy, the inerter is a device that corresponded to the capacitor completely where conquers the nature restriction of mass, what's more, it is significant to improve the ratio of the inerter's inertance to its mass for mechanical networks synthesis. And according to the principle of active-control-based mechatronic inerter, we present two implementation schemes. One was based on linear motor, and the other was based on the ball screw and rotary motor. We introduced the implementation methods and established theoretical model of the two schemes, then compared the ratio of the inerter's inertance to its mass for the two schemes. Finally, we consider the scheme is better which was based on the ball screw and rotary motor.
\end{abstract}

\section{Introduction}

In 2002, the concept of inerter was proposed by Malcolm C. Smith [1]. The inerter is a mechanical device which has two free and independent terminals [2], and the quantitative relation between the force acting on the two terminals and the relative acceleration between the two terminals are as shown in the formula (1).

$$
\mathbf{F}=b\left(\dot{\mathbf{v}}_{\mathbf{1}}-\dot{\mathbf{v}}_{\mathbf{2}}\right)
$$

in which $\mathbf{F}$ is the applied force, while $\mathbf{v}_{\mathbf{1}}$ and $\mathbf{v}_{\mathbf{2}}$ are the velocities of the two terminals. The constant of proportionality $b$ is called the inertance and has units of kilograms [3]. Thus, Inerter is essentially a two terminals mass element. The concept of inerter was put forward, which solved the problem that the mass only as single terminal mass components relative to the inertial system, and the mechanical network and circuit network can be strictly corresponding to the force-current analogy [4]. It has important theory and application value for applying circuit theory to mechanical network synthesis.

The traditional inerter needs to be realized by the mechanical mechanism while enhanced the inertance needs to be increased by the transmission ratio and the mass of the flywheel [5]. However, it will inevitably lead to the increase of the mass of the inerter, and limits the application of the inerter in mass sensitive areas such as aerospace [6]. For this purpose, we put forward the concept of mechatronic inerter, and explore the feasibility of using electromagnetic force to enhance the inertance [7].

The active-control-based mechatronic inerter (hereinafter referred to as ACBMI) are composed of sensors, controllers and actuators. Its working principle is: F was applied to the two terminals of the actuator to make relative motion, while measured by the sensor and sent to the controller; according to set parameters and $\mathbf{F}$, the controller control actuator always hinder the movement of $\mathbf{F}$ that the relative acceleration between the two terminals is reduced, and the inertance is enhanced[ 8]. The schematic diagram is shown in figure 1 .

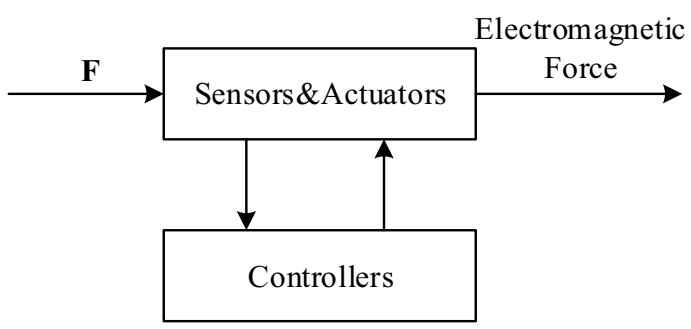

Figure 1. Principle block diagram of ACBMI.

According to different actuator implementation schemes, the ACBMIs are divided into two kinds [9]. One is the use of linear motor as the actuator (hereinafter referred to as the linear motor based ACBMI), $\mathbf{F}$ was applied to the primary and secondary of the linear motor, and linear motor directly generate electromagnetic force to prevent the linear motion caused by applied force; another is the use of ball screw and rotary motor as the actuator (hereinafter referred to as the rotary motor based ACBMI), the ball screw to achieve the conversion between linear motion and rotary motion, and the electromagnetic torque generated by the rotary motor hinders the movement of $\mathbf{F}$.

We built the theoretical model of two kinds of the ACBMIs, and compared the relationship between the 
inertance and the inerter own mass [10]. The ratio between inertance and mass of inerter is defined as RMI (the ratio of mass to intance). The greater RMI of inerter, then the greater inertance can be achieved for the same mass of inerter [11]. Therefore, we can choose a better implementation scheme by comparing RMI.

\section{Theoretical model of two kinds of ACBMIs}

Assuming the mass of two kinds of inerters are both $M$, and building the theoretical model.

\subsection{Theoretical model of linear motor based ACBMI}

The mass of linear motor based ACBMI equal to the mass of linear motor, so the mass of linear motor is $M$. Among them, the mass of primary is $m_{l}$, the mass of secondary was $M_{1}$, and meet $M=m_{1}+M_{1}$.

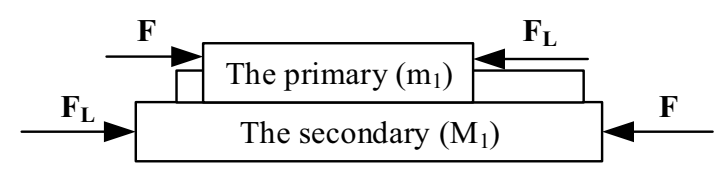

Figure 2. The force diagram of linear motor based ACBMI

As shown in Figure 2, $\mathbf{F}$ applied to the two terminals of linear motor based ACBMI, and the continuous thrust of linear motor output is $\mathbf{F}_{\mathbf{L}}$. Without consideration of friction, the relative acceleration of the two terminals is

$$
\begin{gathered}
\mathbf{a}_{\mathbf{L} 1}=\frac{\left(\mathbf{F}-\mathbf{F}_{\mathbf{L}}\right)}{m_{1}} \\
\mathbf{a}_{\mathbf{L} \mathbf{2}}=\frac{\left(\mathbf{F}-\mathbf{F}_{\mathbf{L}}\right)}{M_{1}} \\
\mathbf{a}_{\mathbf{L}}=\mathbf{a}_{\mathbf{L} \mathbf{1}}+\mathbf{a}_{\mathbf{L} 2} \\
\mathbf{a}_{\mathbf{L}}=\frac{\left(\mathbf{F}-\mathbf{F}_{\mathbf{L}}\right) M}{m_{1}\left(M-m_{1}\right)}
\end{gathered}
$$

in which $\mathbf{a}_{\mathbf{L} 1}$ is the acceleration of primary, $\mathbf{a}_{\mathbf{L} 2}$ is the acceleration of secondary.

The inertance $b_{L}$ and the RMI $g_{L}$ of the linear motor based ACBMI are respectively

$$
\begin{aligned}
& b_{L}=\frac{\mathbf{F}}{\mathbf{a}_{\mathbf{L}}}=\frac{\mathbf{F}}{\left(\mathbf{F}-\mathbf{F}_{\mathbf{L}}\right)} \cdot \frac{m_{1}\left(M-m_{1}\right)}{M} \\
& g_{L}=\frac{b_{L}}{M}=\frac{\mathbf{F}}{\left(\mathbf{F}-\mathbf{F}_{\mathbf{L}}\right)} \cdot \frac{m_{1}\left(M-m_{1}\right)}{M^{2}}
\end{aligned}
$$

\subsection{Theoretical model of rotary motor based ACBMI}

The mass of rotary motor based ACBMI equal to the mass of rotary moto and the mass of ball screw. Let $m_{21}$ be the mass of the ball screw nut, $m_{22}$ the mass of the screw, $r$ the radius of the screw, $p$ the lead of the screw, $J_{1}$ the moment of inertia of rotor, $M_{2}$ the mass of the rotary motor, and $M=m_{21}+m_{22}+M_{2}$.

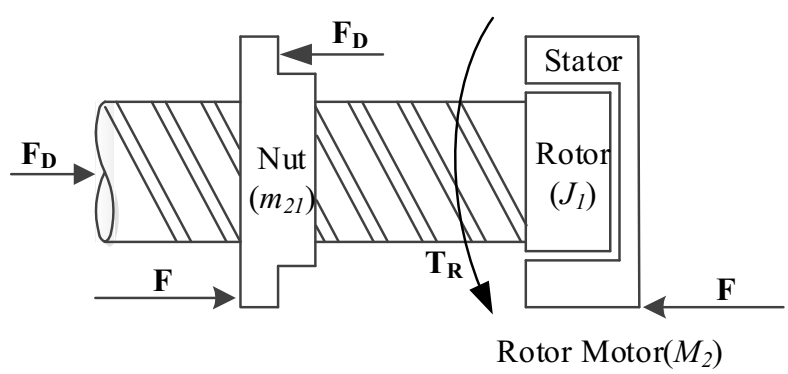

Figure 3. The force diagram of rotor motor based ACBMI

F applied to the two terminals of the linear motor based ACBMI, the output torque of the rotary motor with mass of $M_{2}$ is $\mathbf{T}_{\mathbf{R}}$, the force analysis of the inerter is as shown in figure 3.

Among them, $\mathbf{F}_{\mathbf{D}}$ is the axial force between the nut and the screw, and have the following relationship with the drive torque $\mathbf{T}_{\mathbf{D}}$

$$
\mathbf{F}_{\mathbf{D}}=\frac{2 \pi}{p} \mathbf{T}_{\mathbf{D}}
$$

For the screw and the rotor has the following relationship

$$
\begin{gathered}
J_{2}=\frac{1}{2} m_{22} r^{2} \\
J=J_{1}+J_{2}=J_{1}+\frac{1}{2} m_{22} r^{2} \\
\mathbf{T}_{\mathbf{D}}-\mathbf{T}_{\mathbf{R}}=J \boldsymbol{\beta} \\
\mathbf{a}_{\mathbf{2 1}}=\frac{\mathbf{F}-\mathbf{F}_{\mathbf{D}}}{m_{21}} \\
\mathbf{a}_{\mathbf{2 2}}=\frac{\mathbf{F}-\mathbf{F}_{\mathbf{D}}}{m_{22}+M_{2}} \\
\frac{\mathbf{a}_{\mathbf{2 1}}}{\boldsymbol{\beta}}=\frac{p}{2 \pi} \\
\mathbf{a}_{\mathbf{R}}=\mathbf{a}_{\mathbf{2 1}}+\mathbf{a}_{\mathbf{2 2}}
\end{gathered}
$$

in which $J_{2}$ is the moment of inertia of the screw, $J$ is the moment of inertia of the screw and the rotor, $\boldsymbol{\beta}$ is the angular acceleration of rotating screw and rotor, $\mathbf{a}_{21}$ is the acceleration of nut, $\mathbf{a}_{22}$ is the acceleration of motor, $\mathbf{a}_{\mathbf{R}}$ is the relative acceleration of the two terminals.

Combined $(8) \sim(15)$, then 


$$
\begin{aligned}
& \mathbf{a}_{21}=\frac{\left(\mathbf{F}-\frac{2 \pi}{p} \mathbf{T}_{\mathbf{R}}\right)\left(m_{22}+M_{2}\right)}{\left[m_{21}+\left(\frac{2 \pi}{p}\right)^{2} J_{1}+\frac{1}{2}\left(\frac{2 \pi r}{p}\right)^{2} m_{22}\right]\left(M-m_{21}\right)} \\
& \mathbf{a}_{22}=\frac{\left(\mathbf{F}-\frac{2 \pi}{p} \mathbf{T}_{\mathbf{R}}\right) m_{21}}{\left[m_{21}+\left(\frac{2 \pi}{p}\right)^{2} J_{1}+\frac{1}{2}\left(\frac{2 \pi r}{p}\right)^{2} m_{22}\right]\left(M-m_{21}\right)}
\end{aligned}
$$

And the relative acceleration of the two terminals is

$$
\mathbf{a}_{\mathbf{R}}=\frac{\left(\mathbf{F}-\frac{2 \pi}{p} \mathbf{T}_{\mathbf{R}}\right) M}{\left[m_{21}+\left(\frac{2 \pi}{p}\right)^{2} J_{1}+\frac{1}{2}\left(\frac{2 \pi r}{p}\right)^{2} m_{22}\right]\left(M-m_{21}\right)}
$$

The inertance $b_{R}$ and the RMI $g_{R}$ of the rotary motor based ACBMI are respectively

$$
\begin{aligned}
& b_{R}=\frac{\left[m_{21}+\left(\frac{2 \pi}{p}\right)^{2} J_{1}+\frac{1}{2}\left(\frac{2 \pi r}{p}\right)^{2} m_{22}\right]\left(M-m_{21}\right) \mathbf{F}}{\left(\mathbf{F}-\frac{2 \pi}{p} \mathbf{T}_{\mathbf{R}}\right) M} \\
& g_{R}=\frac{\left[m_{21}+\left(\frac{2 \pi}{p}\right)^{2} J_{1}+\frac{1}{2}\left(\frac{2 \pi r}{p}\right)^{2} m_{22}\right]\left(M-m_{21}\right) \mathbf{F}}{\left(\mathbf{F}-\frac{2 \pi}{p} \mathbf{T}_{\mathbf{R}}\right) M^{2}}
\end{aligned}
$$

\section{Comparison}

\section{1. $g_{\mathrm{Lmax}}<g_{R \max }$}

For the two kinds of inerters, when the actuator output maximum continuous thrust $\mathbf{F}_{\mathbf{L m a x}}$ and maximum continuous torque $\mathbf{T}_{\mathbf{R} \max }$, we can obtain the maximum RMI

$$
\begin{gathered}
g_{L \text { max }}=\frac{\mathbf{F} m_{1}\left(M-m_{1}\right)}{\left(\mathbf{F}-\mathbf{F}_{\mathbf{L} \text { max }}\right) M^{2}} \\
g_{R \text { max }}=\frac{\left[m_{21}+\left(\frac{2 \pi}{p}\right)^{2} J_{1}+\frac{1}{2}\left(\frac{2 \pi r}{p}\right)^{2} m_{22}\right]\left(M-m_{21}\right) \mathbf{F}}{\left(\mathbf{F}-\frac{2 \pi}{p} \mathbf{T}_{\mathbf{R} \text { max }}\right) M^{2}}
\end{gathered}
$$

In order to compare $g_{L \max }$ and $g_{R \max }$, then let

$$
\begin{gathered}
k_{1}=\frac{\mathbf{F}}{\left(\mathbf{F}-\mathbf{F}_{\mathbf{L} \max }\right)} \\
k_{2}=\frac{m_{1}\left(M-m_{1}\right)}{M^{2}}
\end{gathered}
$$

$$
\begin{gathered}
k_{3}=\frac{\mathbf{F}}{\left(\mathbf{F}-\frac{2 \pi}{p} \mathbf{T}_{\mathrm{R} \max }\right)} \\
k_{4}=\frac{\left[m_{21}+\left(\frac{2 \pi}{p}\right)^{2} J_{1}+\frac{1}{2}\left(\frac{2 \pi r}{p}\right)^{2} m_{22}\right]\left(M-m_{21}\right)}{M^{2}}
\end{gathered}
$$

And $k_{1}<k_{3}$, when

$$
p<\frac{2 \pi \mathbf{T}_{\mathbf{R} \max }}{\mathbf{F}_{\mathbf{L m a x}}}
$$

$k_{2 \max }=0.25$, when

$$
m_{1}=\frac{M}{2}
$$

For the rotary motor inerter $m_{21}<\frac{M}{2}, k_{2}<k_{4}$ (the complete derivation to be given by (36) at the top of the next page) when

$$
p<2 \pi \sqrt{\frac{2 J_{1}}{M}}
$$

So $g_{\text {Lmax }}<g_{\text {Rmax }}$, when

$$
p<\frac{2 \pi \mathbf{T}_{\mathrm{R} \max }}{\mathbf{F}_{\mathrm{Lmax}}} \& p<2 \pi \sqrt{\frac{2 J_{1}}{M}}
$$

In order to facilitate the comparison, let

$$
\begin{gathered}
n_{1}=\frac{\mathbf{F}_{\mathbf{L} \max }}{M} \\
n_{2}=\frac{\mathbf{T}_{\mathrm{Rmax}}}{M_{2}} \\
n_{3}=\frac{M_{2}}{M} \\
n_{4}=\frac{J_{1}}{M_{2}}
\end{gathered}
$$

And

$$
p<\frac{2 \pi n_{2} n_{3}}{n_{1}} \& p<2 \pi \sqrt{2 n_{3} n_{4}}
$$

In order to explore whether there is the existence of $p$ to meet the above relationship, we conducted a market research.

Table 1. Market research of linear motor.

\begin{tabular}{|c|c|}
\hline Types & $\mathbf{n}_{\mathbf{1}}$ \\
\hline IL06 & 20.04 \\
\hline IL12 & 23.04 \\
\hline IL18 & 27.81 \\
\hline IL24 & 28.42 \\
\hline ICD05 & 56.69 \\
\hline ICD10 & 61.66 \\
\hline
\end{tabular}


As shown in Table 1, with comparison for the mass of 5 kilograms of linear motor, we found that $n_{l}$ achieve the maximum value of 61.66 while selected the Kollmorgen ICD10 series linear motor. And as shown in Table 2, with comparison for the mass of 5 kilograms of rotary motor, we found that $n_{2}$ achieved the maximum value of 0.91 and $n_{4}$ achieved the maximum value of $4.42 \times 10^{-5}$ while selected the Kollmorgen AKM4 series rotary motor.

$$
k_{4}>\frac{\left[m_{21}+\frac{M}{2}+\frac{1}{2}\left(\frac{2 \pi r}{p}\right)^{2} m_{22}\right]\left(M-m_{21}\right)}{M^{2}}>\frac{\frac{M}{2}\left(M-m_{21}\right)}{M^{2}}>\frac{1}{4}>k_{2}
$$

Table 2. Market research of rotary motor.

\begin{tabular}{|c|c|c|}
\hline Types & $\mathbf{n}_{\mathbf{2}}$ & $\mathbf{n}_{\mathbf{4}}$ \\
\hline AKM1X & 0.53 & $6.11 \times 10^{-6}$ \\
\hline AKM2X & 0.77 & $1.50 \times 10^{-5}$ \\
\hline AKM3X & 0.79 & $2.57 \times 10^{-5}$ \\
\hline AKM4X & 0.91 & $4.42 \times 10^{-5}$ \\
\hline CKM & 0.71 & $3.32 \times 10^{-5}$ \\
\hline
\end{tabular}

And through the research of the ball screw, we found that the mass of ball screw is generally below $0.6 \mathrm{~kg}$ and the lead of the screw are rang from $2 \mathrm{~mm}$ to $30 \mathrm{~mm}$ with the appropriate trip and axial load. When selecting the AKM41 rotary motor(the smallest rotary motor in AKM4 series rotary motor), $n_{3}$ gets the minimum value of 0.803 .

Now we plug $n_{1}, n_{2}, n_{3}, n_{4}$ into formula (35), and get $p<0.0529$, then lead is less than $52.9 \mathrm{~mm}$. The RMI of the rotary motor inerter can be obtained more than the linear motor inerter by properly configuring the rotating motor and ball screw while the existing ball screw can meet this condition obviously.

\subsection{Specific examples}

We used inerter design by using the axis rotation of ball screw motor as the actuator, the following parameters

$$
\begin{aligned}
& M=2.657 \mathrm{~kg}, M_{2}=2 \mathrm{~kg}, \mathbf{T}_{\mathbf{R} \max }=0.96 \mathrm{~N} \cdot \mathrm{m}, \\
& J_{1}=2.86 \times 10^{-4} \mathrm{~kg} \cdot \mathrm{m}^{2}, m_{21}=0.32 \mathrm{~kg}, m_{22}=0.337, \\
& r=7.5 \mathrm{~mm}, p=20 \mathrm{~mm}, \mathbf{F}=400 \mathrm{~N}
\end{aligned}
$$

gave a value of $g_{\text {Rmax }}=39.63$.

If the linear motor is used as the actuator, and the ICD10-050 linear motor with excellent performance is selected. The following parameters

$$
\begin{aligned}
& M=2.8 \mathrm{~kg}, \mathbf{F}_{\mathbf{L m a x}}=171 \mathrm{~N}, \mathbf{F}=400 \mathrm{~N}, \\
& m_{1}=1.9 \mathrm{~kg}, M_{1}=0.9 \mathrm{~kg}
\end{aligned}
$$

gave a value of $g_{\text {Lmax }}=0.39$.

\section{Conclusions}

We built the theoretical model of two kinds of inerters with different actuators, and analyzed the condition when the inertia of the rotary motor based ACBMI outweighed that of the linear motor based ACBMI. Through marker research, we found that the condition was easy to satisfy. According to specific examples, the RMI of the rotary motor inerter was much higher than that of the linear motor inerter. Therefore, the RMI of the inerters can be enhanced by applying ball screw and rotary motor as actuator.

\section{References}

1. M.C. Smith, IEEE Trans. Automatic Control, Synthesis of Mechanical Networks: The Inerter, 47, 1648-1662 (2002)

2. J. Nie, X. Zhang, B. Jiang, L. Chen, Machine Design and Research, Researh on the Inerter Structure, 28, 29-32 (2012)

3. D.X. Zhu, Y.Z. Liu, J.L. Wen, ICIA, A Simulation Research of the Mechatronic Inerter Based on Active Control, (2015)

4. M.C. Smith, F. Wang, IEEE Conference on Decision and Control, Performance Benefits in Passive Vehicle Suspensions Employing Inerters, 28, 29-32 (2012)

5. C. Papageorgiou, N. Houghton, M.C. Smith, Journal of Dynamic Systems Measurement and Control, Experimental Testing and Analysis of Inerter Devices, 131, 3-6 (2009)

6. M.C. Smith, F. Wang, IEEE Conference on Decision and Control, Performance Benefits in Passive Vehicle Suspensions Employing Inerters, 28,29-32 (2012)

7. F. Wang, M. Hong, The Institution of Mechanical Engineers, Designing and testing a hydraulic inerter, 225, 65-72 (2011)

8. L. Chen, X. Yang, R. Wang and Y. Shen, Chinese Journal of Mechanical Engineering, Analysis on Design and Performances of Vehicle Passive Inerterspring-damper Suspension Based on Dynamic Vibration Absorbers, 28, 30-34 (2012)

9. J. Nie, X. Zhang, B. Hu and L. Chen, Vehicle and Power Technology, A New Approach of Vehicle Passive Suspension Techniques, 128, 59-64 (2012)

10. F. Wang and H. Chan, IEEE Conference on Decision and Control, Mechatronic Suspension Design and Its Applications to Vehicle Suspension Control, 3770 (2008)

11. S. Evangelou, D. Limebeer, J. Sharp, and M. Smith, IEEE Control Systems Magazine, Control of Motorcycle Steering Instabilities, 10, 81-85 (2006) 